\title{
Toward Developing a Standardized Core Set of Outcome Measures in Mobile Health Interventions for Tuberculosis Management: Systematic Review
}

Seohyun Lee ${ }^{1}$, PhD; Youngji Lee ${ }^{2}$, MA; Sangmi Lee ${ }^{2}$, MPH; Sheikh Mohammed Shariful Islam ${ }^{3}$, PhD; Sun-Young $\mathrm{Kim}^{2}, \mathrm{PhD}$

\footnotetext{
${ }^{1}$ Institute of Health \& Environment, Center for Global Health Research, Graduate School of Public Health, Seoul National University, Seoul, Republic of Korea

${ }^{2}$ Department of Public Health Sciences, Graduate School of Public Health, Seoul National University, Seoul, Republic of Korea

${ }^{3}$ Institute for Physical Activity and Nutrition, School of Exercise and Nutrition Sciences, Faculty of Health, Deakin University, Melbourne, Australia
}

\section{Corresponding Author:}

Sun-Young Kim, PhD

Department of Public Health Sciences

Graduate School of Public Health

Seoul National University

1 Gwanak-ro, Gwanak-gu

Seoul, 08826

Republic of Korea

Phone: 828802768

Fax: 8227622888

Email: sykim22@snu.ac.kr

\begin{abstract}
Background: Tuberculosis (TB) management can be challenging in low- and middle-income countries (LMICs) not only because of its high burden but also the prolonged treatment period involving multiple drugs. With rapid development in mobile technology, mobile health (mHealth) interventions or using a mobile device for TB management has gained popularity. Despite the potential usefulness of mHealth interventions for TB, few studies have quantitatively synthesized evidence on its effectiveness, presumably because of variability in outcome measures reported in the literature.
\end{abstract}

Objective: The aim of this systematic review was to evaluate the outcome measures reported in TB mHealth literature in LMICs.

Methods: MEDLINE, EMBASE, and the Cochrane Database of Systematic Reviews were searched to identify mHealth intervention studies for TB (published up to May 2018) that reported any type of outcome measures. The extracted information included the study setting, types of mHealth technology used, target population, study design, and categories of outcome measures. Outcomes were classified into 13 categories including treatment outcome, adherence, process measure, perception, technical outcome, and so on. The qualitative synthesis of evidence focused on the categories of outcome measures reported by the type of mHealth interventions.

Results: A total of 27 studies were included for the qualitative synthesis of evidence. The study designs varied widely, ranging from randomized controlled trials to economic evaluations. A total of 12 studies adopted short message service (SMS), whereas 5 studies used SMS in combination with additional technologies or mobile apps. The study populations were also diverse, including patients with TB, patients with TB/HIV, health care workers, and general patients attending a clinic. There was a wide range of variations in the definition of outcome measures across the studies. Among the diverse categories of outcome measures, treatment outcomes have been reported in 14 studies, but only 6 of them measured the outcome according to the standard TB treatment definitions by the World Health Organization.

Conclusions: This critical evaluation of outcomes reported in mHealth studies for TB management suggests that substantial variability exists in reporting outcome measures. To overcome the challenges in evidence synthesis for mHealth interventions, this study can provide insights into the development of a core set of outcome measures by intervention type and study design.

(JMIR Mhealth Uhealth 2019;7(2):e12385) doi: $\underline{10.2196 / 12385}$ 


\section{KEYWORDS}

mHealth; tuberculosis; outcome measures; evidence synthesis; low-and middle-income countries

\section{Introduction}

Tuberculosis (TB) is one of the deadly infectious diseases that have claimed millions of lives worldwide. According to the World Health Organization (WHO), globally, there were 10.4 million new TB cases causing approximately 1.2 million deaths in 2016 [1,2]. The mortality rate of TB is disproportionately higher in low- and middle-income countries (LMICs). Over 95\% of TB deaths occurred in these countries, and 7 LMICs (India, Indonesia, China, Philippines, Pakistan, Nigeria, and South Africa) accounted for $64 \%$ of the total burden [3]. In fact, previous studies have shown that there is empirical evidence of positive associations between poverty indicators and TB incidence both at the macro and individual levels [4]. Considering the vicious cycle of poverty and TB, alleviating the burden of TB is more challenging for the LMICs because it requires adequate resources for "prolonged treatment with multiple drugs [5]." The 6-month course of first-line therapy can be burdensome with the possibility of adverse reactions and the treatment of multidrug-resistant (MDR)-TB requires more toxic and expensive drugs [6]. For example, the cost of bedaquiline, a second-line medication to treat MDR-TB, was US $\$ 3000$ per treatment in middle-income countries and US $\$ 900$ in low-income countries [7]. In fact, premature discontinuation of the treatment, which can lead to MDR-TB, is common among TB patients not only for its toxicity but also for socioeconomic costs associated with it [8]. Therefore, the management of TB is notoriously difficult especially in LMICs.

In this context, using mobile devices for TB treatment has been recognized as an innovative approach for LMICs where mobile subscription rates have dramatically increased over the past decade. Mobile health (mHealth) interventions involving mobile devices in the management of TB have the potential for reducing costs of information delivery and improving the quality of communication [9]. mHealth can be useful for TB treatment adherence support such as short message service (SMS) for medication reminders or mobile apps for remote directly observed treatment (DOT) strategy [10,11].

Despite the potential of mHealth interventions for improving TB management, the empirical evidence on its effectiveness is mixed. Some studies have demonstrated the effectiveness and feasibility of the mHealth interventions for TB [12,13], whereas others have shown no significant impact $[14,15]$. Moreover, no study has attempted to synthesize the results quantitatively to rigorously evaluate the effectiveness of mHealth in TB management. Presumably, one of the reasons for such difficulty in synthesizing and evaluating the findings comes from wide variations in the outcomes reported from the mHealth studies for TB.

To respond to this knowledge gap, this study aimed to systematically review previous mHealth studies for TB management and critically evaluate and categorize the outcome measures for different mobile technologies and study designs. The goal of this study was to provide researchers insights into the development of a core set of outcome measures for mHealth interventions intended to improve TB treatment adherence. In doing so, the study can facilitate the evidence synthesis of mHealth interventions for TB.

\section{Methods}

\section{Search Strategy and Review Process}

Electronic databases (MEDLINE, EMBASE, and Cochrane Database of Systematic Reviews) were searched to identify peer-reviewed studies of mHealth interventions for TB. The systematic search was supplemented by reviewing relevant review papers identified from the initial search. The search strategy for the study population includes key terms describing LMICs such as "resource poor" or "developing country." The search strategy for the mHealth intervention combined multiple keywords such as "mHealth" and "text-messaging." As for the target disease, "tuberculosis," "TB," "multi-drug resistant tuberculosis," and "MDR-TB" were used as search terms. No restrictions were applied to the publication type or publication date, but the language filter was applied to identify studies published in English. The search included articles published up to May 2018. The full search strategy is available in Multimedia Appendix 1.

Furthermore, 3 authors (ShL, YL, and SmL) independently reviewed the retrieved studies throughout the selection process. Each study identified from the databases was screened by 2 reviewers and then a full-text review was conducted for the potentially eligible studies. The disagreement on the selection process was resolved by the other authors who were not involved in the review of the specific study under discussion.

\section{Eligibility for Review}

The inclusion criteria for this systematic review were as follows: First, studies conducted in the context of LMICs, as defined by the World Bank's income cutoffs [16]; second, studies involving an intervention using mobile devices (ie, mHealth intervention); third, the target disease of the study should be TB or MDR-TB; fourth, studies designed to evaluate the effectiveness or benefits of mHealth interventions for TB (eg, observational study, mixed-methods study or implementation project, randomized controlled trial [RCT]); fifth, studies reporting more than one type of outcome; finally, only full-text studies published in English were considered eligible. In addition, the authors attempted to identify individual studies from reviews or systematic reviews, which were included in this study.

\section{Data Extraction and Analysis}

The qualitative synthesis of evidence focused on the outcome measures reported in each type of mHealth intervention. Information about the study setting, mHealth technologies used, target populations, and types of outcome measures was extracted. To classify the diverse types of detailed outcome measures, the following categories were used: (1) treatment outcome; (2) treatment outcome as defined by WHO; (3) adherence; (4) process measure; (5) perception; (6) technical 
outcome; (7) health outcome; (8) quality of life; (9) knowledge; (10) cost-effectiveness; (11) cost; (12) psychosocial outcome; and (13) mortality. Some explanations about these measures are provided in the next few paragraphs.

The treatment outcome includes any outcome measure that deals with the result of TB treatment, such as sputum smear conversion or microscopy test result. The treatment outcome following the WHO definition was separately categorized [17]. The WHO definition was developed to make a distinction for treatment outcomes between the drug-susceptible TB and drug-resistant $\mathrm{TB}$, which are mutually exclusive groups. According to the WHO definition, any patient with TB should belong to either group and then 1 of the 7 treatment outcome cohorts: (1) cured; (2) treatment completed; (3) treatment failed; (4) died; (5) lost to follow-up; (6) not evaluated; and (7) treatment success. The WHO definitions for each of these 7 categories differ between the drug-susceptible TB and drug-resistant TB as described in Multimedia Appendix 2.

The adherence outcome includes medication adherence or treatment adherence. The process measure is any outcome measure related to treatment process, including the receipt of diagnostic test, attendance to appointments, or reporting of adverse events. The perception indicates any outcome measure that captures the user's thoughts on mHealth for TB management. The technical outcome relates to the outcome measures that investigate the technical feasibility such as processing times or system installation. Health outcome, quality of life, knowledge (eg, patients' understanding of the disease or the technology), cost-effectiveness (ie, the extent to which an alternative provides value for money), cost (ie, costs associated with an intervention from different perspectives), psychosocial outcome, and mortality outcomes are additional categories which are self-explanatory.

\section{Risk of Bias: Quality Assessment}

To evaluate the quality of individual studies included for our review, risk of bias was assessed with the existing tools. As this systematic review includes various types of studies, it is important to have a coherent set of quality assessment tools for different study designs. Therefore, we used the modified version of the Critical Appraisal Skills Program (CASP) that provides the checklists specific to various types of studies ranging from RCTs and qualitative studies to economic evaluation [18]. In case of mixed-methods studies whose CASP tool has not been developed yet, the quality assessment criteria for mixed-methods studies from the previous study were employed [19]. The quality assessment of studies is presented in Multimedia Appendix 3.

\section{Results}

\section{Overview of Included Studies}

Among the 312 studies identified after removing duplicates, 260 articles were excluded during the screening process based on the titles and abstracts. Therefore, 52 articles were assessed for eligibility through a full-text review. Of those, 27 studies were included for the qualitative synthesis of evidence. A flow diagram for the selection process, based on the Preferred
Reporting Items for Systematic Reviews and Meta-Analyses guidelines, is provided in Figure 1 [20].

\section{Qualitative Synthesis of Evidence}

Table 1 presents the results of qualitative synthesis of the mHealth studies for TB management. Approximately, half (15 out of 27) of the studies were conducted in African countries. The study designs were diverse, including 6 RCTs, 5 mixed-methods studies, 1 cohort study, 3 qualitative studies, 4 observational studies, 6 implementation projects, and 2 economic evaluation studies. The types of mHealth technologies utilized were diverse as well: 12 studies employed SMS, 6 studies used mobile app, 5 studies used SMS plus other technology, 3 studies utilized phone calls, and only 1 study applied mHealth for mobile data collection. With regard to the study population, a majority (20 out of 27 ) of the studies were targeted for TB patients or TB/HIV patients, but there were a few studies that examined the experience of health care workers or general patients at the clinic for TB test results notification.

In terms of the outcome measures, there was a wide range of variants in their definitions even within each category of the outcomes. For instance, both Mohammed et al and Bediang et al defined treatment success as the primary outcome measure of SMS intervention for TB medication adherence in their RCT, but they defined treatment success differently [15,22]. Mohammed et al defined it as "the sum of patients clinically reported as cured (ie, a patient whose sputum smear or culture was positive at the beginning of treatment but who was smearor culture-negative in the last month of treatment and on at least one previous occasion) or treatment completed (ie, a patient who completed treatment but who does not have a negative sputum smear or culture result in the last month of treatment and on at least one previous occasion)" [22]. On the contrary, Bediang et al defined treatment success as "having completed 6 months treatment and having negative sputum smears at 5 months" [15].

\section{Summary of Outcome Measures}

Table 2 summarizes the types of outcome measures reported in the included studies, using the categories defined in this review. The most frequently reported outcome type was treatment success. Approximately half of the studies reviewed (14 out of 27 ) included treatment outcome but only 6 studies among them followed the WHO definition for the treatment outcome (Multimedia Appendix 2). The second most frequently reported category was perception on the mHealth intervention (13 out of 27). Other categories reported were diverse and included technical outcome, medication or treatment adherence, process measure, etc. However, there was substantial variability within each category of outcome, as shown in Table 1. For example, acceptability and satisfaction within the perception category were defined differently from one study to another [15,28-30,32,35,36,40,44]. Also, SMS-only intervention studies did not focus on technical outcome [12,15,22,29,30,32,35, $36,42,43,45,46]$ whereas studies involving other mHealth technologies such as an app or mobile data collection did so $[21,28,33,37,39-41]$. On the contrary, outcomes related to cost or cost-effectiveness were reported only via studies involving SMS $[29,45,46]$. 
Figure 1. Flow diagram for selection process following the Preferred Reporting Items for Systematic Reviews and Meta-Analyses (PRISMA) guideline. mHealth: mobile health; TB: tuberculosis.

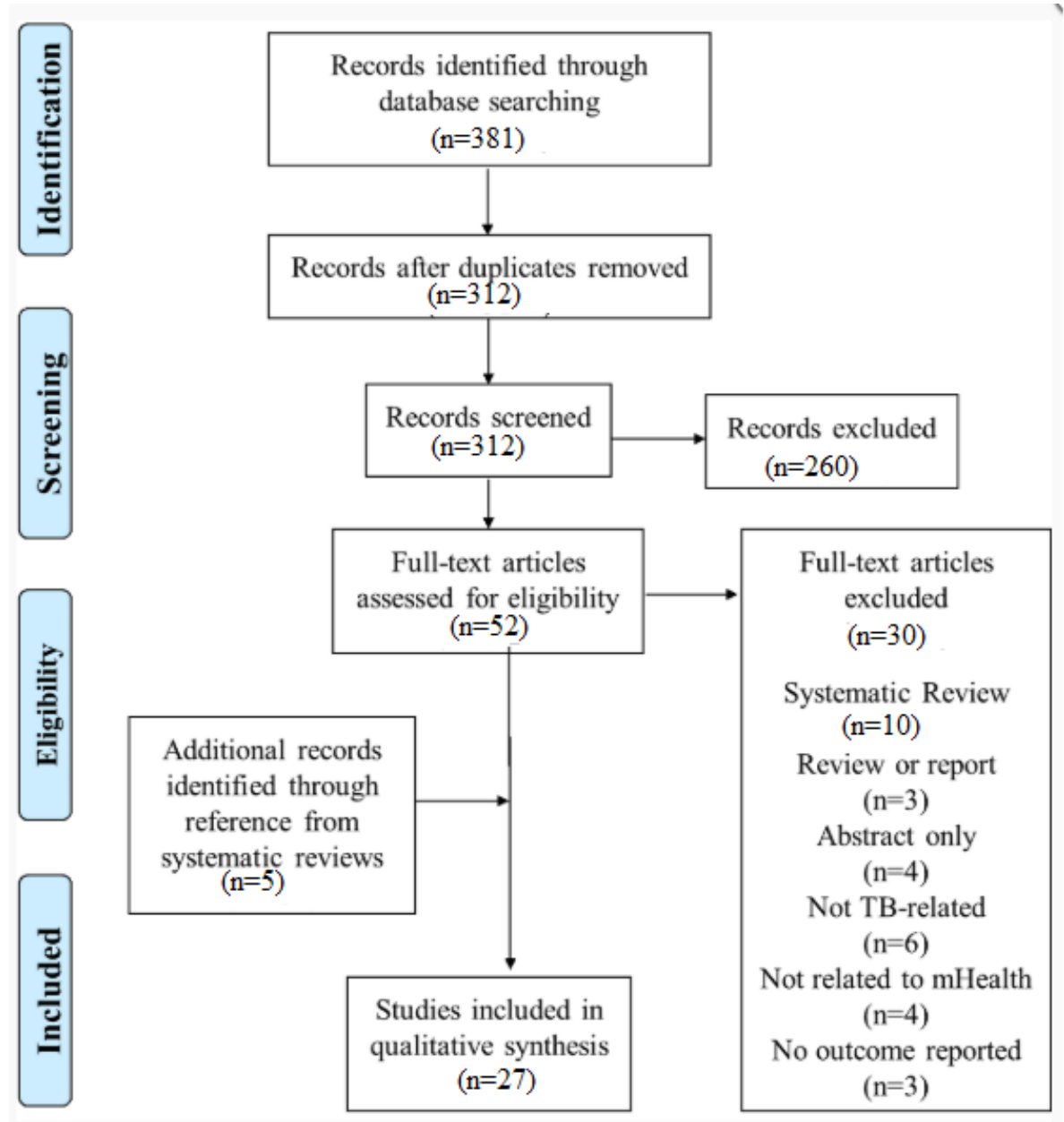


Table 1. Summary of included studies.

\begin{tabular}{|c|c|c|c|c|c|c|c|}
\hline Source & Country & Study design & $\begin{array}{l}\text { Mobile } \\
\text { health tech- } \\
\text { nology }\end{array}$ & Population & Outcome measures & $\begin{array}{l}\text { Outcome } \\
\text { category }\end{array}$ & Purpose \\
\hline $\begin{array}{l}\text { Blaya, } 2009 \\
{[21]}\end{array}$ & Peru & $\mathrm{RCT}^{\mathrm{a}}$ & $\begin{array}{l}\text { Mobile data } \\
\text { collection }\end{array}$ & $\begin{array}{l}\text { Health cen- } \\
\text { ters }\end{array}$ & $\begin{array}{l}\text { Processing times, frequency of errors, the } \\
\text { number of work-hours expended by data } \\
\text { collectors }\end{array}$ & $\begin{array}{l}\text { Technical } \\
\text { outcome }\end{array}$ & $\begin{array}{l}\text { For laborato- } \\
\text { ry data col- } \\
\text { lection }\end{array}$ \\
\hline $\begin{array}{l}\text { Mohammed, } \\
2016 \text { [22] }\end{array}$ & Pakistan & RCT & $\mathrm{SMS}^{\mathrm{b}}$ & $\mathrm{TB}^{\mathrm{C}}$ patients & $\begin{array}{l}\text { Primary: clinically recorded treatment suc- } \\
\text { cess based upon intention-to-treat; Sec- } \\
\text { ondary: treatment outcomes (WHO defini- } \\
\text { tions }{ }^{\mathrm{d}} \text { ), self-reported medication adherence, } \\
\text { self-reported psychological and physical } \\
\text { health measures }\end{array}$ & $\begin{array}{l}\text { Treatment } \\
\text { outcome, ad- } \\
\text { herence, } \\
\text { health out- } \\
\text { come }\end{array}$ & $\begin{array}{l}\text { For medica- } \\
\text { tion adher- } \\
\text { ence }\end{array}$ \\
\hline $\begin{array}{l}\text { Bassett, } \\
2013[23] \\
\text { Bassett, } \\
2016[24]\end{array}$ & South Africa & $\begin{array}{l}\text { RCT proto- } \\
\text { col (2013); } \\
\text { RCT (2016) }\end{array}$ & $\begin{array}{l}\text { SMS and } \\
\text { phone calls }\end{array}$ & $\begin{array}{l}\text { Patients at } \\
\text { clinic }\end{array}$ & $\begin{array}{l}\text { Primary: treatment completion; Secondary: } \\
\text { mortality, receipt of CD4 count and TB test } \\
\text { results, and repeat CD4 counts for those not } \\
\text { antiretroviral therapy (ART)-eligible at } \\
\text { baseline }\end{array}$ & $\begin{array}{l}\text { Treatment } \\
\text { outcome, } \\
\text { mortality, } \\
\text { process mea- } \\
\text { sure }\end{array}$ & $\begin{array}{l}\text { For appoint- } \\
\text { ment and } \\
\text { test result re- } \\
\text { minder and } \\
\text { psychosocial } \\
\text { support }\end{array}$ \\
\hline $\begin{array}{l}\text { Huang, } 2017 \\
{[25]}\end{array}$ & China & $\begin{array}{l}\text { Cluster RCT } \\
\text { protocol }\end{array}$ & Mobile app & TB patients & $\begin{array}{l}\text { Primary: TB treatment result (WHO defini- } \\
\text { tions }{ }^{\mathrm{d}} \text { ); Secondary: treatment adherence } \\
\text { (the percentage of patients receiving TB } \\
\text { treatment who missed fewer than } 5 \% \text { of } \\
\text { doses), self-reported adherence, knowledge } \\
\text { about TB, quality of life (QoL) }\end{array}$ & $\begin{array}{l}\text { Treatment } \\
\text { outcome, ad- } \\
\text { herence, } \\
\text { knowledge, } \\
\text { QoL }\end{array}$ & $\begin{array}{l}\text { For Bracelet- } \\
\text { and self-di- } \\
\text { rected obser- } \\
\text { vational ther- } \\
\text { apy }\end{array}$ \\
\hline $\begin{array}{l}\text { Bediang, } \\
2014[26] \\
\text { Bediang, } \\
2018[15]\end{array}$ & Cameroon & $\begin{array}{l}\text { RCT proto- } \\
\text { col (2014); } \\
\text { RCT (2018) }\end{array}$ & SMS & TB patients & $\begin{array}{l}\text { Primary: cure rate (absence of Koch's } \\
\text { bacilli in the sputum), treatment success } \\
\text { (having completed } 6 \text { months' treatment and } \\
\text { having negative sputum smears at } 5 \\
\text { months); Secondary: treatment adherence } \\
\text { (drug prescriptions collected and doses } \\
\text { taken), attendance to appointments, punctu- } \\
\text { ality of appointments, treatment outcome } \\
\text { (WHO definitions }{ }^{\mathrm{d}} \text { ), the number of partici- } \\
\text { pants who develop resistance, satisfaction }\end{array}$ & $\begin{array}{l}\text { Treatment } \\
\text { outcome, ad- } \\
\text { herence, pro- } \\
\text { cess mea- } \\
\text { sure, percep- } \\
\text { tion }\end{array}$ & $\begin{array}{l}\text { For medica- } \\
\text { tion adher- } \\
\text { ence }\end{array}$ \\
\hline $\begin{array}{l}\text { Khachadouri- } \\
\text { an, } 2015 \\
{[27]}\end{array}$ & Armenia & $\begin{array}{l}\text { RCT proto- } \\
\text { col }\end{array}$ & $\begin{array}{l}\text { SMS and } \\
\text { phone calls }\end{array}$ & TB patients & $\begin{array}{l}\text { Primary: physician-reported treatment out- } \\
\text { come (WHO definitions }{ }^{\mathrm{d}} \text { ); Secondary: pa- } \\
\text { tients' knowledge, depression, QoL, within- } \\
\text { family TB-related stigma, family social } \\
\text { support, self-reported treatment adherence }\end{array}$ & $\begin{array}{l}\text { Treatment } \\
\text { outcome, } \\
\text { knowledge, } \\
\text { psychosocial } \\
\text { outcome, } \\
\text { QoL, adher- } \\
\text { ence }\end{array}$ & $\begin{array}{l}\text { For medica- } \\
\text { tion adher- } \\
\text { ence }\end{array}$ \\
\hline $\begin{array}{l}\text { Chaiyachati, } \\
2013 \text { [28] }\end{array}$ & South Africa & $\begin{array}{l}\text { Mixed-meth- } \\
\text { ods study }\end{array}$ & Mobile app & $\begin{array}{l}\text { Health care } \\
\text { workers }\end{array}$ & $\begin{array}{l}\text { Primary: proportion of weekly adverse } \\
\text { events forms submitted vs expected by mo- } \\
\text { bile health care workers; Secondary: accept- } \\
\text { ability (perceived comfort levels with using } \\
\text { mobile phone technology), quality of ad- } \\
\text { verse events monitoring, proportion of re- } \\
\text { portable adverse events being captured; } \\
\text { Technical outcomes: phone usage patterns, } \\
\text { technical problems experienced }\end{array}$ & $\begin{array}{l}\text { Process mea- } \\
\text { sure, percep- } \\
\text { tion, techni- } \\
\text { cal outcome }\end{array}$ & $\begin{array}{l}\text { For adverse } \\
\text { events report- } \\
\text { ing }\end{array}$ \\
\hline $\begin{array}{l}\text { Howard, } \\
2016 \text { [29] }\end{array}$ & Lesotho & $\begin{array}{l}\text { Mixed-meth- } \\
\text { ods, cluster- } \\
\text { randomized } \\
\text { trial protocol }\end{array}$ & SMS & $\begin{array}{l}\text { TB/HIV pa- } \\
\text { tients, health } \\
\text { care workers }\end{array}$ & $\begin{array}{l}\text { Primary: ART initiation, retention, and TB } \\
\text { treatment success; Secondary: time to ART } \\
\text { initiation, adherence, change in cluster of } \\
\text { differentiation } 4 \text { (CD4) count, sputum smear } \\
\text { conversion, cost-effectiveness, acceptability }\end{array}$ & $\begin{array}{l}\text { Process mea- } \\
\text { sure, treat- } \\
\text { ment out- } \\
\text { come, adher- } \\
\text { ence, cost-ef- } \\
\text { fectiveness, } \\
\text { perception }\end{array}$ & $\begin{array}{l}\text { For treat- } \\
\text { ment adher- } \\
\text { ence }\end{array}$ \\
\hline
\end{tabular}




\begin{tabular}{|c|c|c|c|c|c|c|c|}
\hline Source & Country & Study design & $\begin{array}{l}\text { Mobile } \\
\text { health tech- } \\
\text { nology }\end{array}$ & Population & Outcome measures & $\begin{array}{l}\text { Outcome } \\
\text { category }\end{array}$ & Purpose \\
\hline $\begin{array}{l}\text { Iribarren, } \\
2013 \text { [30] }\end{array}$ & Argentina & $\begin{array}{l}\text { Mixed-meth- } \\
\text { ods study } \\
\text { (including } \\
\text { RCT) }\end{array}$ & SMS & TB patients & $\begin{array}{l}\text { Primary: feasibility (access to mobile } \\
\text { phones, familiarity with texting, rate of } \\
\text { participant refusal, suboptimal TB under- } \\
\text { standing), and acceptability (feeling cared } \\
\text { for patient's treatment, self-reporting adher- } \\
\text { ence); Secondary: initial efficacy (mi- } \\
\text { croscopy test result from positive to nega- } \\
\text { tive, treatment outcome) }\end{array}$ & $\begin{array}{l}\text { Process mea- } \\
\text { sure, percep- } \\
\text { tion, adher- } \\
\text { ence, treat- } \\
\text { ment out- } \\
\text { come }\end{array}$ & $\begin{array}{l}\text { For treat- } \\
\text { ment adher- } \\
\text { ence }\end{array}$ \\
\hline $\begin{array}{l}\text { Hirsch- } \\
\text { Moverman, } \\
\text { 2017a [31] }\end{array}$ & $\begin{array}{l}\text { Lesotho, } \\
\text { Ethiopia }\end{array}$ & $\begin{array}{l}\text { Mixed-meth- } \\
\text { ods; imple- } \\
\text { mentation } \\
\text { science } \\
\text { study }\end{array}$ & Phone calls & $\begin{array}{l}\text { TB/HIV pa- } \\
\text { tients, } \\
\text { TB patients }\end{array}$ & $\begin{array}{l}\text { The number of call attempts per participant } \\
\text { for each month, completeness of monthly } \\
\text { calls, success rates, challenges }\end{array}$ & $\begin{array}{l}\text { Process mea- } \\
\text { sure }\end{array}$ & $\begin{array}{l}\text { For medica- } \\
\text { tion adher- } \\
\text { ence }\end{array}$ \\
\hline $\begin{array}{l}\text { Hirsch- } \\
\text { Moverman, } \\
\text { 2017b [32] }\end{array}$ & Lesotho & $\begin{array}{l}\text { Mixed-meth- } \\
\text { ods imple- } \\
\text { mentation } \\
\text { science } \\
\text { study, clus- } \\
\text { ter-random- } \\
\text { ized trial } \\
\text { protocol }\end{array}$ & SMS & $\begin{array}{l}\text { TB patients, } \\
\text { health care } \\
\text { workers, } \\
\text { caregivers }\end{array}$ & $\begin{array}{l}\text { Primary: the number of child contacts Isoni- } \\
\text { azid preventive therapy (IPT) initiation, IPT } \\
\text { completion; Secondary: HIV testing, yield } \\
\text { of active prevalent TB among child con- } \\
\text { tacts, acceptability, and utilization of com- } \\
\text { munity-based intervention components }\end{array}$ & $\begin{array}{l}\text { Treatment } \\
\text { outcome, } \\
\text { perception, } \\
\text { process mea- } \\
\text { sure }\end{array}$ & $\begin{array}{l}\text { For medica- } \\
\text { tion adher- } \\
\text { ence and ap- } \\
\text { pointment } \\
\text { reminders }\end{array}$ \\
\hline $\begin{array}{l}\text { Nguyen, } \\
2017 \text { [33] }\end{array}$ & Vietnam & Cohort study & $\begin{array}{l}\text { SMS and } \\
\text { mobile app }\end{array}$ & TB patients & $\begin{array}{l}\text { Primary: proportion of patients completing } \\
\text { all doses of self-administered treatment; } \\
\text { Secondary: proportion of videos uploaded } \\
\text { as scheduled, proportion of patients discon- } \\
\text { tinuing using Video DOT (VDOT) }\end{array}$ & $\begin{array}{l}\text { Treatment } \\
\text { outcome, } \\
\text { technical } \\
\text { outcome, } \\
\text { process mea- } \\
\text { sure }\end{array}$ & $\begin{array}{l}\text { For medica- } \\
\text { tion adher- } \\
\text { ence }\end{array}$ \\
\hline $\begin{array}{l}\text { Daftary, } \\
2017[34]\end{array}$ & Ethiopia & $\begin{array}{l}\text { Qualitative } \\
\text { study }\end{array}$ & $\begin{array}{l}\text { Interactive } \\
\text { voice re- } \\
\text { sponse } \\
\text { (IVR) }\end{array}$ & HIV patients & $\begin{array}{l}\text { Perceptions and attitude, perceived benefits } \\
\text { and challenges }\end{array}$ & Perception & $\begin{array}{l}\text { For preven- } \\
\text { tive therapy } \\
\text { adherence }\end{array}$ \\
\hline $\begin{array}{l}\text { Albino, } 2014 \\
{[35]}\end{array}$ & Peru & $\begin{array}{l}\text { Qualitative } \\
\text { study }\end{array}$ & SMS & TB patients & Perceptions and acceptability & Perception & $\begin{array}{l}\text { For treat- } \\
\text { ment adher- } \\
\text { ence }\end{array}$ \\
\hline $\begin{array}{l}\text { Nhavoto, } \\
2017 \text { [36] }\end{array}$ & Mozambique & $\begin{array}{l}\text { Qualitative } \\
\text { study }\end{array}$ & SMS & $\begin{array}{l}\text { TB patients, } \\
\text { Health care } \\
\text { workers }\end{array}$ & $\begin{array}{l}\text { Usefulness, perceived benefits, ease of use, } \\
\text { satisfaction, risks of the SMS system }\end{array}$ & Perception & $\begin{array}{l}\text { For treat- } \\
\text { ment adher- } \\
\text { ence }\end{array}$ \\
\hline $\begin{array}{l}\text { Hoffman, } \\
2010[37]\end{array}$ & Kenya & $\begin{array}{l}\text { Observation- } \\
\text { al study }\end{array}$ & Mobile app & TB patients & $\begin{array}{l}\text { Primary: technical feasibility (patient and } \\
\text { health provider receptivity to remote direct- } \\
\text { ly observed treatment [DOT]); Secondary: } \\
\text { patient preferences and receptivity to receiv- } \\
\text { ing TB health message on a mobile phone }\end{array}$ & $\begin{array}{l}\text { Technical } \\
\text { outcome, } \\
\text { perception }\end{array}$ & $\begin{array}{l}\text { For Mobile } \\
\text { Direct Obser- } \\
\text { vation of } \\
\text { Treatment }\end{array}$ \\
\hline $\begin{array}{l}\text { de Sumari- } \\
\text { de Boer, } \\
2016[12]\end{array}$ & Tanzania & $\begin{array}{l}\text { Observation- } \\
\text { al pilot study }\end{array}$ & SMS & $\begin{array}{l}\text { HIV pa- } \\
\text { tients, TB } \\
\text { patients }\end{array}$ & $\begin{array}{l}\text { Quantitative: percentage of doses taken on } \\
\text { time, percentage of sent reminders (divided } \\
\text { by total intake prescription), percentage of } \\
\text { correct reminders (after missed doses), per- } \\
\text { centage of incorrect reminders (after open- } \\
\text { ing the pillbox but the signal was not sent), } \\
\text { percentage of extra openings, percentage of } \\
\text { missed doses, percentage of adherence with } \\
\text { the exclusion of doses that were taken after } \\
\text { a reminder; Qualitative: general experience } \\
\text { with using the device }\end{array}$ & $\begin{array}{l}\text { Process mea- } \\
\text { sure, adher- } \\
\text { ence, percep- } \\
\text { tion }\end{array}$ & $\begin{array}{l}\text { For medica- } \\
\text { tion adher- } \\
\text { ence }\end{array}$ \\
\hline $\begin{array}{l}\text { Garfein, } \\
2015[38]\end{array}$ & $\begin{array}{l}\text { Mexico, } \\
\text { USA }\end{array}$ & $\begin{array}{l}\text { Observation- } \\
\text { al pilot study }\end{array}$ & Mobile app & TB patients & $\begin{array}{l}\text { Primary: adherence rate (the number of } \\
\text { medication doses observed in videos divided } \\
\text { by the number of doses expected during the } \\
\text { treatment period); Secondary: perceptions } \\
\text { of VDOT }\end{array}$ & $\begin{array}{l}\text { Adherence, } \\
\text { perception }\end{array}$ & For VDOT \\
\hline
\end{tabular}




\begin{tabular}{|c|c|c|c|c|c|c|c|}
\hline Source & Country & Study design & $\begin{array}{l}\text { Mobile } \\
\text { health tech- } \\
\text { nology }\end{array}$ & Population & Outcome measures & $\begin{array}{l}\text { Outcome } \\
\text { category }\end{array}$ & Purpose \\
\hline $\begin{array}{l}\text { Dwolatzky, } \\
2006 \text { [39] }\end{array}$ & South Africa & $\begin{array}{l}\text { Observation- } \\
\text { al pilot study }\end{array}$ & Mobile app & $\begin{array}{l}\text { Patients at } \\
\text { clinic }\end{array}$ & Time taken to locate the households & $\begin{array}{l}\text { Technical } \\
\text { outcome }\end{array}$ & $\begin{array}{l}\text { For locating } \\
\text { patients' } \\
\text { homes by } \\
\text { global posi- } \\
\text { tioning sys- } \\
\text { tem and per- } \\
\text { sonal digital } \\
\text { assistant }\end{array}$ \\
\hline $\begin{array}{l}\mathrm{Ha}, 2016 \\
{[40]}\end{array}$ & Botswana & $\begin{array}{l}\text { Implementa- } \\
\text { tion project }\end{array}$ & Mobile app & TB patients & $\begin{array}{l}\text { Cases screened for contact tracing, time re- } \\
\text { quired to complete TB contact tracing per } \\
\text { contact, quality of data collected, user satis- } \\
\text { faction with usability, operational consider- } \\
\text { ations }\end{array}$ & $\begin{array}{l}\text { Technical } \\
\text { outcome, } \\
\text { perception }\end{array}$ & $\begin{array}{l}\text { For contact } \\
\text { tracing }\end{array}$ \\
\hline $\begin{array}{l}\text { Cowan, } \\
2016[41]\end{array}$ & Mozambique & $\begin{array}{l}\text { Implementa- } \\
\text { tion project }\end{array}$ & $\begin{array}{l}\text { SMS and } \\
\text { mobile app }\end{array}$ & $\begin{array}{l}\text { Health facili- } \\
\text { ty }\end{array}$ & $\begin{array}{l}\text { System installation on computers, develop- } \\
\text { ment of Web-based interface and automated } \\
\text { SMS and email messages, test results upload- } \\
\text { ed to the system, SMS notifications sent to } \\
\text { key personnel, the number of users }\end{array}$ & $\begin{array}{l}\text { Technical } \\
\text { outcome }\end{array}$ & $\begin{array}{l}\text { For remote } \\
\text { monitoring } \\
\text { solution }\end{array}$ \\
\hline $\begin{array}{l}\mathrm{Ku}- \\
\text { nawararak, } \\
2011[14]\end{array}$ & Thailand & $\begin{array}{l}\text { Implementa- } \\
\text { tion project }\end{array}$ & Phone calls & TB patients & $\begin{array}{l}\text { Cure rates, completion rates, failure rates } \\
\text { and success rates, conversion rates }\end{array}$ & $\begin{array}{l}\text { Treatment } \\
\text { outcome }\end{array}$ & $\begin{array}{l}\text { For medica- } \\
\text { tion adher- } \\
\text { ence }\end{array}$ \\
\hline $\begin{array}{l}\text { Lorent, } 2014 \\
\text { [42] }\end{array}$ & Cambodia & $\begin{array}{l}\text { Implementa- } \\
\text { tion project }\end{array}$ & SMS & $\begin{array}{l}\text { General pop- } \\
\text { ulation }\end{array}$ & $\begin{array}{l}\text { TB case detection- smear-positivity, clinical } \\
\text { TB treatment uptake-time to treatment ini- } \\
\text { tiation outcome-treatment outcomes (WHO } \\
\text { definitions }^{\mathrm{d}} \text { ), delay in linkage to care }\end{array}$ & $\begin{array}{l}\text { Process mea- } \\
\text { sure, treat- } \\
\text { ment out- } \\
\text { come }\end{array}$ & $\begin{array}{l}\text { For test re- } \\
\text { sult notifica- } \\
\text { tion }\end{array}$ \\
\hline $\begin{array}{l}\text { Mahmud, } \\
2010[43]\end{array}$ & Malawi & $\begin{array}{l}\text { Implementa- } \\
\text { tion project }\end{array}$ & SMS & $\begin{array}{l}\text { Health care } \\
\text { workers }\end{array}$ & $\begin{array}{l}\text { Operational net savings, worker time } \\
\text { gained, patient enrollment }\end{array}$ & $\begin{array}{l}\text { Process mea- } \\
\text { sure }\end{array}$ & $\begin{array}{l}\text { For continu- } \\
\text { ity of care }\end{array}$ \\
\hline $\begin{array}{l}\text { Narasimhan, } \\
2014[44]\end{array}$ & India & $\begin{array}{l}\text { Implementa- } \\
\text { tion project }\end{array}$ & $\begin{array}{l}\text { SMS and } \\
\text { phone calls }\end{array}$ & TB patients & $\begin{array}{l}\text { Treatment completion and cure rates (WHO } \\
\text { definitions }{ }^{\mathrm{d}} \text { ), treatment adherence rates, } \\
\text { adverse drug reaction rates, stigma associat- } \\
\text { ed with TB, patient satisfaction, usage of } \\
\text { the mHealth initiative }\end{array}$ & $\begin{array}{l}\text { Treatment } \\
\text { outcome, ad- } \\
\text { herence, per- } \\
\text { ception }\end{array}$ & $\begin{array}{l}\text { For medica- } \\
\text { tion adher- } \\
\text { ence }\end{array}$ \\
\hline $\begin{array}{l}\text { Broomhead, } \\
2012[45]\end{array}$ & South Africa & $\begin{array}{l}\text { Cost mini- } \\
\text { mization } \\
\text { analysis }\end{array}$ & SMS & TB patients & $\begin{array}{l}\text { Smear conversion rate, TB cure rate, re- } \\
\text { duced average cost per patient }\end{array}$ & $\begin{array}{l}\text { Treatment } \\
\text { outcome, } \\
\text { cost }\end{array}$ & $\begin{array}{l}\text { For treat- } \\
\text { ment adher- } \\
\text { ence }\end{array}$ \\
\hline $\begin{array}{l}\text { Hun- } \\
\text { changsith, } \\
2012 \text { [46] }\end{array}$ & Thailand & $\begin{array}{l}\text { Cost-effec- } \\
\text { tive-ness } \\
\text { analysis }\end{array}$ & SMS & TB patients & $\begin{array}{l}\text { Disability-adjusted life years (DALYs) } \\
\text { averted, costs (health care perspective), ef- } \\
\text { fects of interventions, success rate, failure } \\
\text { rate, transfer out rate, death rate }\end{array}$ & $\begin{array}{l}\text { Health out- } \\
\text { come, treat- } \\
\text { ment out- } \\
\text { come, cost, } \\
\text { cost-effec- } \\
\text { tive-ness }\end{array}$ & $\begin{array}{l}\text { For medica- } \\
\text { tion adher- } \\
\text { ence }\end{array}$ \\
\hline
\end{tabular}

${ }^{\mathrm{a}} \mathrm{RCT}$ : randomized controlled trial.

${ }^{\mathrm{b}} \mathrm{SMS}$ : short message service.

${ }^{\mathrm{c}}$ TB: tuberculosis.

${ }^{\mathrm{d}}$ World Health Organization definitions: presented in Multimedia Appendix 2. 
Table 2. Reported outcomes by mHealth intervention type.

\begin{tabular}{|c|c|c|c|c|c|c|c|c|c|c|c|c|c|}
\hline \multirow{2}{*}{$\begin{array}{l}\text { Intervention } \\
\text { type (number } \\
\text { of studies) and } \\
\text { reference }\end{array}$} & \multicolumn{13}{|c|}{ Categories of outcome measure } \\
\hline & $\begin{array}{l}\text { Treat- } \\
\text { ment } \\
\text { out- } \\
\text { come }\end{array}$ & $\begin{array}{l}\text { Treat- } \\
\text { ment out- } \\
\text { come by } \\
\mathrm{WHO}^{\mathrm{a}} \\
\text { definition }\end{array}$ & $\begin{array}{l}\text { Adher- } \\
\text { ence }\end{array}$ & $\begin{array}{l}\text { Process } \\
\text { measure }\end{array}$ & $\begin{array}{l}\text { Percep- } \\
\text { tion }\end{array}$ & $\begin{array}{l}\text { Techni- } \\
\text { cal out- } \\
\text { come }\end{array}$ & $\begin{array}{l}\text { Health } \\
\text { out- } \\
\text { come }\end{array}$ & $\mathrm{QoL}^{\mathrm{b}}$ & $\begin{array}{l}\text { Knowl- } \\
\text { edge }\end{array}$ & $\begin{array}{l}\text { Cost-ef- } \\
\text { fective- } \\
\text { ness }\end{array}$ & Cost & $\begin{array}{l}\text { Psychoso- } \\
\text { cial out- } \\
\text { come }\end{array}$ & Mortality \\
\hline
\end{tabular}

\section{Short message service (SMS; 12 studies)}

[22]

\section{SMS plus others (5 studies)}
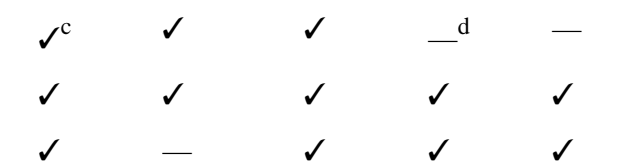

Mobile app (6 studies)

$\begin{array}{lllllllllllllll}{[25]} & \checkmark & \checkmark & \checkmark & - & - & - & - & \checkmark & \checkmark & - & - & - & - \\ {[28]} & - & - & - & \checkmark & \checkmark & \checkmark & - & - & - & - & - & - & - \\ {[37]} & - & - & - & - & \checkmark & \checkmark & - & - & - & - & - & - & - \\ {[38]} & - & - & \checkmark & - & \checkmark & - & - & - & - & - & - & - & - \\ {[39]} & - & - & - & - & - & \checkmark & - & - & - & - & - & - & - \\ {[40]} & - & - & - & - & \checkmark & \checkmark & - & - & - & - & - & - & -\end{array}$

Phone calls or interactive voice response (3 studies)

Mobile data collection (1 study)

$$
\begin{array}{cccccc}
- & - & - & \checkmark & - & - \\
- & - & - & - & \checkmark & - \\
\checkmark & - & - & - & - & -
\end{array}
$$$$
\begin{array}{lllllll}
- & - & - & - & - & - & - \\
- & - & - & - & - & - & - \\
- & - & - & - & - & - & -
\end{array}
$$

${ }^{\mathrm{a}}$ WHO: World Health Organization.

${ }^{\mathrm{b}} \mathrm{QoL}$ : quality of life.

${ }^{\mathrm{c}}$ Tick marks indicate that the specific category of outcome measure was reported.

${ }^{\mathrm{d}}$ Outcome measure was not reported. 


\section{Discussion}

\section{Principal Findings}

This systematic review critically evaluated the outcomes reported in mHealth studies for TB management in LMICs. The reason why rigorous evidence synthesis is warranted is that recent literature for $\mathrm{TB}$ reports mixed results despite the rapid implementation of mHealth technology for TB management. The fragmented pieces of evidence on the effectiveness partly resulted from the wide variations in the definitions of outcome measures in TB mHealth interventions. Even though treatment outcome has been reported by many studies, they often did not adopt the standard definition recommended by the WHO [17].

The WHO definition of TB treatment outcome is part of an effort to standardize outcome measures for $\mathrm{TB}$ at the global level. To promote the use of standardized sets of outcome measures for TB, WHO provided the standard definitions and classifications of TB in terms of diagnosis or treatment outcomes [17]. The intention for this standardization effort was to coordinate international comparison of TB treatment outcomes through health information systems. However, the findings of our review revealed that mHealth studies for TB have not comprehensively adopted this standardized approach for TB treatment. Only 6 out of 27 interventions chose to report the treatment outcome according to the WHO definition. Interventions involving phone calls, interactive voice response, or mobile data collection did not consider the WHO definition.

Our findings also suggest that, to rigorously evaluate the effectiveness of mHealth interventions for TB, future studies should be carefully designed with regard to the selection of outcome measures. Indeed, using standard definitions for outcome measures within some commonly reported categories can improve comparability across different studies. As assessed in this study, examples of such categories include treatment outcome (preferably using the WHO definition), perception, process measure, adherence, and technical outcome.

The value of this systematic review can be found in its potential to motivate and facilitate consensus on standard definitions of outcome measures used in mHealth interventions for TB so that such effort can guide more effective mHealth intervention designs for improving TB management. Although current literature shows considerable variability in the definition of outcome measures, discussion and coordination among researchers can promote standardized methods in measuring outcomes. Specifically, the outcomes should be comparable, promote transparent communication, and maintain consistency in terminology. Coordination at the global level is necessary to develop a core set of outcome measures for TB mHealth interventions by study design and technology type utilized.

When choosing a core set of outcome measures with standard definitions, there are additional issues to consider. First, the time point for reporting outcome measures should be clinically meaningful and feasible [47]. Second, a detailed description of the measure should be provided, such as calculation method or definitions. Third, a clear explanation on the target population for each outcome measure can be useful. For example, some outcome measures may be more appropriate for MDR-TB patients rather than TB patients on their first-line therapy course. Finally, long-term outcome measures should be considered to establish fundamental evidence for TB mHealth interventions. The long-term outcomes can be related to physical, psychosocial, or mental health. Despite its importance, our review showed that only 2 out of 27 studies reported long-term health outcomes; Mohammed et al, reported self-reported psychological and physical health measures [22] and Hunchangsith et al, reported DALYs averted [46].

Another issue to consider is related to evidence for cost. As this study suggested, insufficient evidence exists in terms of cost-effectiveness or cost of mHealth interventions for TB management. Those previous studies that have evaluated the cost-effectiveness or cost of mHealth interventions only considered SMS as mHealth channels and did not consider or evaluate other mHealth channels and technologies. However, other mHealth channels and technologies such as mobile apps or global positioning system are now available for TB patients [48]. Therefore, future studies need to assess the cost-effectiveness of such channels and technologies for improving TB management in LMICs.

This study has some limitations. First, the database used for identifying relevant studies is limited to the 3 most frequently cited sources, namely MEDLINE, EMBASE, and Cochrane Database of Systematic Reviews. Grey literature or other sources of information can supplement our findings. To complement this limitation, we attempted to identify additional related studies from relevant systematic reviews searched from our study. Second, the effectiveness of the mHealth interventions for TB was not quantitatively evaluated because of the heterogeneity of the outcomes reported.

Despite these limitations, this study provides an overview of the currently reported outcome measures for mHealth interventions intended to improve TB management in the context of LMICs. The results from this review can be used as a starting point for discussion to adopt standardized definitions within different categories of outcome measures for future mHealth interventions for TB management in LMICs.

\section{Conclusions}

This systematic review of mHealth studies for TB suggests that substantial variability exists with regard to the definitions of outcome measures across studies. Our review highlights that a standardized method for measuring the different outcomes is warranted to improve comparability of outcome measures across studies for a more rigorous and reliable evaluation of the effectiveness of mHealth interventions for TB. In doing so, the coordination among researchers and the development of a core set of outcome measures based on standardized methods would be necessary. Our study provides useful information for researchers to better assess the effectiveness of mHealth interventions for TB. In addition, the study provides insights into the possibility of developing a core set of outcome measures by intervention type and study design based on a standardized or coordinated set of methods. 


\section{Acknowledgments}

ShL was involved in the conceptualization of the study and drafted the manuscript. ShL, YL, and SmL developed the search strategy, collected data, and reviewed the identified studies. SMSI critically reviewed the manuscript. SYK critically reviewed the manuscript and interpreted the findings. All authors reviewed and approved the final version of the manuscript. This work was supported by the National Research Foundation of Korea Grant funded by the Korean Government (\#21B20151213037).

\section{Conflicts of Interest}

None declared.

\section{Multimedia Appendix 1}

Search strategy.

[PDF File (Adobe PDF File), 129KB-Multimedia Appendix 1]

\section{Multimedia Appendix 2}

Definitions of TB treatment outcome by the WHO [17].

[PDF File (Adobe PDF File), 34KB-Multimedia Appendix 2]

\section{Multimedia Appendix 3}

Risk of bias assessment.

[PDF File (Adobe PDF File), 794KB-Multimedia Appendix 3]

\section{References}

1. World Health Organization. Global Health Observatory (GHO) Data URL: http://www.who.int/gho/tb/en/ [accessed 2018-11-20] [WebCite Cache ID 743tX88Tq]

2. Institute for Health Metrics and Evaluation. GBD Results Tool URL: http://ghdx.healthdata.org/gbd-results-tool [accessed 2018-11-20] [WebCite Cache ID 743u1Lk8z]

3. World Health Organization. Tuberculosis URL: http://www.who.int/news-room/fact-sheets/detail/tuberculosis [accessed 2018-11-20] [WebCite Cache ID 743u9swGr]

4. Oxlade O, Murray M. Tuberculosis and poverty: why are the poor at greater risk in India? PLoS One 2012 Nov;7(11):e47533 [FREE Full text] [doi: 10.1371/journal.pone.0047533] [Medline: 23185241]

5. Roberts D, Rylands J, Sinclair D. Interventions using mobile devices (phones, smart phones, or tablets) to improve adherence to treatment for HIV or tuberculosis. Cochrane Database Syst Rev 2016 Sep 15;9. [doi: 10.1002/14651858.CD012353]

6. Nagarajan S, Whitaker P. Management of adverse reactions to first-line tuberculosis antibiotics. Curr Opin Allergy Clin Immunol 2018 Aug;18(4):333-341. [doi: 10.1097/ACI.0000000000000462] [Medline: 29901473]

7. Wirtz VJ, Hogerzeil HV, Gray AL, Bigdeli M, de Joncheere CP, Ewen MA, et al. Essential medicines for universal health coverage. Lancet 2017 Jan 28;389(10067):403-476. [doi: 10.1016/S0140-6736(16)31599-9] [Medline: 27832874]

8. Babiarz KS, Suen SC, Goldhaber-Fiebert JD. Tuberculosis treatment discontinuation and symptom persistence: an observational study of Bihar, India's public care system covering >100,000,000 inhabitants. BMC Public Health 2014 May 01;14:418 [FREE Full text] [doi: 10.1186/1471-2458-14-418] [Medline: 24886314]

9. Aranda-Jan CB, Mohutsiwa-Dibe N, Loukanova S. Systematic review on what works, what does not work and why of implementation of mobile health (mHealth) projects in Africa. BMC Public Health 2014 Feb 21;14:188 [FREE Full text] [doi: 10.1186/1471-2458-14-188] [Medline: 24555733]

10. Story A, Garfein RS, Hayward A, Rusovich V, Dadu A, Soltan V, et al. Monitoring therapy compliance of tuberculosis patients by using video-enabled electronic devices. Emerg Infect Dis 2016 Mar;22(3):538-540 [FREE Full text] [doi: 10.3201/eid2203.151620] [Medline: 26891363]

11. Ngwatu BK, Nsengiyumva NP, Oxlade O, Mappin-Kasirer B, Nguyen NL, Jaramillo E, Collaborative group on the impact of digital technologies on TB. The impact of digital health technologies on tuberculosis treatment: a systematic review. Eur Respir J 2018 Jan;51(1) [FREE Full text] [doi: 10.1183/13993003.01596-2017] [Medline: 29326332]

12. de Sumari-de Boer IM, van den Boogaard J, Ngowi KM, Semvua HH, Kiwango KW, Aarnoutse RE, et al. Feasibility of real time medication monitoring among HIV infected and TB patients in a resource-limited setting. AIDS Behav 2016 May;20(5):1097-1107. [doi: 10.1007/s10461-015-1254-0] [Medline: 26604004]

13. Devi BR, Syed-Abdul S, Kumar A, Iqbal U, Nguyen PA, Li YC, et al. mHealth: an updated systematic review with a focus on HIV/AIDS and tuberculosis long term management using mobile phones. Comput Methods Programs Biomed 2015 Nov;122(2):257-265. [doi: 10.1016/j.cmpb.2015.08.003] [Medline: 26304621] 
14. Kunawararak P, Pongpanich S, Chantawong S, Pokaew P, Traisathit P, Srithanaviboonchai K, et al. Tuberculosis treatment with mobile-phone medication reminders in northern Thailand. Southeast Asian J Trop Med Public Health 2011 Nov;42(6):1444-1451. [Medline: 22299414]

15. Bediang G, Stoll B, Elia N, Abena JL, Geissbuhler A. SMS reminders to improve adherence and cure of tuberculosis patients in Cameroon (TB-SMS Cameroon): a randomised controlled trial. BMC Public Health 2018 May 2;18(1):583 [FREE Full text] [doi: 10.1186/s12889-018-5502-x] [Medline: 29720146]

16. World Bank. World Bank Country and Lending Groups URL: https://datahelpdesk.worldbank.org/knowledgebase/articles/ 906519-world-bank-country-and-lending-groups [accessed 2018-11-20] [WebCite Cache ID 743uHlrLZ]

17. World Health Organization. Definitions and reporting framework for tuberculosis-2013 revision URL: https://www.who.int/ tb/publications/definitions/en/ [accessed 2019-01-14] [WebCite Cache ID 75Qsg5qy4]

18. Critical Appraisal Skills Programme. CASP Checklist URL: https://casp-uk.net/casp-tools-checklists/ [accessed 2018-12-03] [WebCite Cache ID 74NiKvDGi]

19. Atkins S, Launiala A, Kagaha A, Smith H. Including mixed methods research in systematic reviews: examples from qualitative syntheses in TB and malaria control. BMC Med Res Methodol 2012 Apr 30;12:62 [FREE Full text] [doi: 10.1186/1471-2288-12-62] [Medline: 22545681]

20. Moher D, Liberati A, Tetzlaff J, Altman DG, PRISMA group. Preferred reporting items for systematic reviews and meta-analyses: the PRISMA statement. PLoS Med 2009 Jul 21;6(7):e1000097 [FREE Full text] [doi:

10.1371/journal.pmed.1000097] [Medline: 19621072]

21. Blaya JA, Cohen T, Rodríguez P, Kim J, Fraser HS. Personal digital assistants to collect tuberculosis bacteriology data in Peru reduce delays, errors, and workload, and are acceptable to users: cluster randomized controlled trial. Int J Infect Dis 2009 May;13(3):410-418 [FREE Full text] [doi: 10.1016/j.ijid.2008.09.015] [Medline: 19097925]

22. Mohammed S, Glennerster R, Khan AJ. Impact of a daily SMS medication reminder system on Tuberculosis treatment outcomes: a randomized controlled trial. PLoS One 2016 Nov 1;11(11):e0162944 [FREE Full text] [doi: 10.1371/journal.pone.0162944] [Medline: 27802283]

23. Bassett IV, Giddy J, Chaisson CE, Ross D, Bogart LM, Coleman SM, et al. A randomized trial to optimize HIV/TB care in South Africa: design of the Sizanani trial. BMC Infect Dis 2013 Aug 26;13:390 [FREE Full text] [doi: 10.1186/1471-2334-13-390] [Medline: 23972276]

24. Bassett IV, Coleman SM, Giddy J, Bogart LM, Chaisson CE, Ross D, et al. Sizanani: a randomized trial of health system navigators to improve linkage to HIV and TB care in South Africa. J Acquir Immune Defic Syndr 2016 Oct 01;73(2):154-160 [FREE Full text] [doi: 10.1097/QAI.0000000000001025] [Medline: 27632145]

25. Huang R, Ren G, Hu J. Bracelet- and self-directed observational therapy for control of tuberculosis: study protocol for a cluster randomized controlled trial. Trials 2017 Jul 4;18(1):286 [FREE Full text] [doi: 10.1186/s13063-017-1996-2] [Medline: 28673323]

26. Bediang G, Stoll B, Elia N, Abena JL, Nolna D, Chastonay P, et al. SMS reminders to improve the tuberculosis cure rate in developing countries (TB-SMS Cameroon): a protocol of a randomised control study. Trials 2014 Jan 24;15:35 [FREE Full text] [doi: $\underline{10.1186 / 1745-6215-15-35}$ ] [Medline: $\underline{24460827}$ ]

27. Khachadourian V, Truzyan N, Harutyunyan A, Thompson ME, Harutyunyan T, Petrosyan V. People-centered tuberculosis care versus standard directly observed therapy: study protocol for a cluster randomized controlled trial. Trials 2015 Jun 22;16:281 [FREE Full text] [doi: 10.1186/s13063-015-0802-2] [Medline: 26093675]

28. Chaiyachati KH, Loveday M, Lorenz S, Lesh N, Larkan LM, Cinti S, et al. A pilot study of an mHealth application for healthcare workers: poor uptake despite high reported acceptability at a rural South African community-based MDR-TB treatment program. PLoS One 2013 May 28;8(5):e64662 [FREE Full text] [doi: 10.1371/journal.pone.0064662] [Medline: 23724075]

29. Howard AA, Hirsch-Moverman Y, Frederix K, Daftary A, Saito S, Gross T, et al. The START Study to evaluate the effectiveness of a combination intervention package to enhance antiretroviral therapy uptake and retention during TB treatment among TB/HIV patients in Lesotho: rationale and design of a mixed-methods, cluster-randomized trial. Glob Health Action 2016;9:31543 [FREE Full text] [doi: 10.3402/gha.v9.31543] [Medline: 27357074]

30. Iribarren S, Beck S, Pearce PF, Chirico C, Etchevarria M, Cardinale D, et al. TextTB: a mixed method pilot study evaluating acceptance, feasibility, and exploring initial efficacy of a text messaging intervention to support TB treatment adherence. Tuberc Res Treat 2013;2013:349394 [FREE Full text] [doi: 10.1155/2013/349394] [Medline: 24455238]

31. Hirsch-Moverman Y, Burkot C, Saito S, Frederix K, Pitt B, Melaku Z, et al. Reaching the end of the line: operational issues with implementing phone-based unannounced pill counts in resource-limited settings. PLoS One 2017 Oct 19;12(10):e0185549 [FREE Full text] [doi: 10.1371/journal.pone.0185549] [Medline: 29049382]

32. Hirsch-Moverman Y, Howard AA, Frederix K, Lebelo L, Hesseling A, Nachman S, et al. The PREVENT study to evaluate the effectiveness and acceptability of a community-based intervention to prevent childhood tuberculosis in Lesotho: study protocol for a cluster randomized controlled trial. Trials 2017 Nov 21;18(1):552 [FREE Full text] [doi: 10.1186/s13063-017-2184-0] [Medline: 29157275] 
33. Nguyen TA, Pham MT, Nguyen TL, Nguyen VN, Pham DC, Nguyen BH, et al. Video directly observed therapy to support adherence with treatment for tuberculosis in Vietnam: a prospective cohort study. Int J Infect Dis 2017 Dec;65:85-89 [FREE Full text] [doi: 10.1016/j.ijid.2017.09.029] [Medline: 29030137]

34. Daftary A, Hirsch-Moverman Y, Kassie GM, Melaku Z, Gadisa T, Saito S, et al. A qualitative evaluation of the acceptability of an interactive voice response system to enhance adherence to Isoniazid preventive therapy among people living with HIV in Ethiopia. AIDS Behav 2017 Nov. [doi: 10.1007/s10461-016-1432-8] [Medline: 27221743]

35. Albino S, Tabb KM, Requena D, Egoavil M, Pineros-Leano MF, Zunt JR, et al. Perceptions and acceptability of short message services technology to improve treatment adherence amongst tuberculosis patients in Peru: a focus group study. PLoS One 2014 May 14;9(5):e95770 [FREE Full text] [doi: 10.1371/journal.pone.0095770] [Medline: 24828031]

36. Nhavoto JA, Grönlund A, Klein GO. Mobile health treatment support intervention for HIV and tuberculosis in Mozambique: perspectives of patients and healthcare workers. PLoS One 2017 Apr 18;12(4):e0176051 [FREE Full text] [doi: 10.1371/journal.pone.0176051] [Medline: 28419149]

37. Hoffman JA, Cunningham JR, Suleh AJ, Sundsmo A, Dekker D, Vago F, et al. Mobile direct observation treatment for tuberculosis patients: a technical feasibility pilot using mobile phones in Nairobi, Kenya. Am J Prev Med 2010 Jul;39(1):78-80. [doi: 10.1016/j.amepre.2010.02.018] [Medline: 20537846]

38. Garfein RS, Collins K, Muñoz F, Moser K, Cerecer-Callu P, Raab F, et al. Feasibility of tuberculosis treatment monitoring by video directly observed therapy: a binational pilot study. Int J Tuberc Lung Dis 2015 Sep;19(9):1057-1064 [FREE Full text] [doi: 10.5588/ijtld.14.0923] [Medline: 26260824]

39. Dwolatzky B, Trengove E, Struthers H, McIntyre JA, Martinson NA. Linking the global positioning system (GPS) to a personal digital assistant (PDA) to support tuberculosis control in South Africa: a pilot study. Int J Health Geogr 2006 Aug 16;5:34 [FREE Full text] [doi: 10.1186/1476-072X-5-34] [Medline: 16911806]

40. Ha YP, Tesfalul MA, Littman-Quinn R, Antwi C, Green R, Mapila TO, et al. Evaluation of a mobile health approach to Tuberculosis contact tracing in Botswana. J Health Commun 2016 Oct;21(10):1115-1121 [FREE Full text] [doi: 10.1080/10810730.2016.1222035] [Medline: 27668973]

41. Cowan J, Michel C, Manhiça I, Mutaquiha C, Monivo C, Saize D, et al. Remote monitoring of Xpert@ MTB/RIF testing in Mozambique: results of programmatic implementation of GxAlert. Int J Tuberc Lung Dis 2016 Mar;20(3):335-341. [doi: 10.5588/ijtld.15.0535] [Medline: 27046714]

42. Lorent N, Choun K, Thai S, Kim T, Huy S, Pe R, et al. Community-based active tuberculosis case finding in poor urban settlements of Phnom Penh, Cambodia: a feasible and effective strategy. PLoS One 2014 Mar;9(3):e92754 [FREE Full text] [doi: 10.1371/journal.pone.0092754] [Medline: 24675985]

43. Mahmud N, Rodriguez J, Nesbit J. A text message-based intervention to bridge the healthcare communication gap in the rural developing world. Technol Health Care 2010;18(2):137-144. [doi: 10.3233/THC-2010-0576] [Medline: 20495253]

44. Narasimhan P, Bakshi A, Kittusami S, Prashant S, Mathai D, Bakshi K, et al. A customized m-Health system for improving Tuberculosis treatment adherence and follow-up in south India. Health Technol 2013 Dec 18;4(1):1-10. [doi: $10.1007 / \mathrm{s} 12553-013-0067-2]$

45. Broomhead S, Mars M. Retrospective return on investment analysis of an electronic treatment adherence device piloted in the Northern Cape Province. Telemed J E Health 2012;18(1):24-31. [doi: 10.1089/tmj.2011.0143] [Medline: 22150713]

46. Hunchangsith P, Barendregt JJ, Vos T, Bertram M. Cost-effectiveness of various tuberculosis control strategies in Thailand. Value Health 2012;15(1 Suppl):S50-S55 [FREE Full text] [doi: 10.1016/j.jval.2011.11.006] [Medline: 22265067]

47. Needham DM. Understanding and improving clinical trial outcome measures in acute respiratory failure. Am J Respir Crit Care Med 2014 Apr 15;189(8):875-877 [FREE Full text] [doi: 10.1164/rccm.201402-0362ED] [Medline: 24735026]

48. Bakibinga P, Kamande E, Omuya M, Ziraba AK, Kyobutungi C. The role of a decision-support smartphone application in enhancing community health volunteers' effectiveness to improve maternal and newborn outcomes in Nairobi, Kenya: quasi-experimental research protocol. BMJ Open 2017 Jul 20;7(7):e014896 [FREE Full text] [doi: 10.1136/bmjopen-2016-014896] [Medline: 28729309]

\section{Abbreviations}

DALY: disability-adjusted life years

DOT: directly observed treatment

IPT: isoniazid preventive therapy

IVR: interactive voice response

LMICs: low- and middle-income countries

MDR-TB: multidrug resistant-TB

mHealth: mobile health

RCT: randomized controlled trial

SMS: short message service

TB: tuberculosis

WHO: World Health Organization 
Edited by G Eysenbach; submitted 02.10.18; peer-reviewed by A Martinez-Millana, A Gray; comments to author 11.11.18; revised version received 03.12.18; accepted 09.12.18; published 19.02.19

Please cite as:

Lee S, Lee Y, Lee S, Islam SMS, Kim SY

Toward Developing a Standardized Core Set of Outcome Measures in Mobile Health Interventions for Tuberculosis Management: Systematic Review

JMIR Mhealth Uhealth 2019;7(2):e12385

URL: http://mhealth.jmir.org/2019/2/e12385/

doi: $10.2196 / 12385$

PMID: 30777847

(CSeohyun Lee, Youngji Lee, Sangmi Lee, Sheikh Mohammed Shariful Islam, Sun-Young Kim. Originally published in JMIR Mhealth and Uhealth (http://mhealth.jmir.org), 19.02.2019. This is an open-access article distributed under the terms of the Creative Commons Attribution License (https://creativecommons.org/licenses/by/4.0/), which permits unrestricted use, distribution, and reproduction in any medium, provided the original work, first published in JMIR mhealth and uhealth, is properly cited. The complete bibliographic information, a link to the original publication on http://mhealth.jmir.org/, as well as this copyright and license information must be included. 